\title{
Adaptive Lyapunov-Based Control of a Robot and Mass-Spring System Undergoing an Impact Collision
}

\author{
Keith Dupree, Student Member, IEEE, Chien-Hao Liang, Student Member, IEEE, \\ Guoqiang Hu, Member, IEEE, and Warren E. Dixon, Senior Member, IEEE
}

\begin{abstract}
The control of dynamic systems that undergo an impact collision is both theoretically challenging and of practical importance. An appeal of studying systems that undergo an impact is that short-duration effects such as high stresses, rapid dissipation of energy, and fast acceleration and deceleration may be achieved from low-energy sources. However, colliding systems present a difficult control challenge because the equations of motion are different when the system suddenly transitions from a noncontact state to a contact state. In this paper, an adaptive nonlinear controller is designed to regulate the states of two dynamic systems that collide. The academic example of a planar robot colliding with an unactuated mass-spring system is used to represent a broader class of such systems. The control objective is defined as the desire to command a robot to collide with an unactuated system and regulate the mass to a desired compressed state while compensating for the unknown constant system parameters. Lyapunov-based methods are used to develop a continuous adaptive controller that yields asymptotic regulation of the mass and robot links. It is interesting to note that one controller is responsible for achieving the control objective when the robot is in free motion (i.e., decoupled from the mass-spring system), when the systems collide, and when the system dynamics are coupled.
\end{abstract}

Index Terms-Adaptive control, backstepping, impact dynamics.

\section{INTRODUCTION}

$\mathbf{R}$ OBOT motion and force control has been well studied for several decades (e.g., [1]-[17]). Most of this paper involves designing a controller to position the end-effector of a robot manipulator constrained to move along a surface to a desired point while controlling the force exerted on the surface. Several techniques to compensate for uncertainty in

Manuscript received August 27, 2007; revised October 30, 2007 and January 8,2008 . This work was supported in part by the National Science Foundation under CAREER Award 0547448, in part by the Air Force Office of Scientific Research under Contract F49620-03-1-0170, in part by Research Grant US-3715-05 from the United States-Israel Binational Agricultural Research and Development Fund, and in part by the Department of Energy (DOE) through Grant DE-FG04-86NE37967 as part of the DOE University Research Program in Robotics. This paper was recommended by Associate Editor H. Gao.

K. Dupree, C.-H. Liang, and W. E. Dixon are with the Department of Mechanical and Aerospace Engineering, University of Florida, Gainesville, FL 32611 USA (e-mail: kdupree@ufl.edu; chliang@ufl.edu; wdixon@ufl.edu).

G. Hu was with the Department of Mechanical and Aerospace Engineering, University of Florida, Gainesville, FL 32611 USA. He is now with the Department of Mechanical and Nuclear Engineering, Kansas State University, Manhattan, KS 66506 USA (e-mail: gqhu@ufl.edu).

Color versions of one or more of the figures in this paper are available online at http://ieeexplore.ieee.org.

Digital Object Identifier 10.1109/TSMCB.2008.923154 the robot dynamics during contact with the surface have also been reported (e.g., [5], [6], [11], [13], and [17]). The robot typically is required to stay in contact with a static surface; however, techniques have also been developed for the robot to remain in contact with a moving object (e.g., [7]-[9]). However, the results from this literature have not addressed the problem where the robot transitions from a noncontact state to an impact collision with another dynamic system, with an objective to then control the coupled dynamic systems.

The control of dynamic systems that undergo an impact collision is both theoretically challenging and of practical importance. If the impact dynamics are not properly modeled and controlled, the impact forces could result in poor system performance and instabilities. One difficulty in controlling systems subject to impacts is that the dynamics are different when the system status transitions from a noncontact state to a contact state. Another difficulty is measuring the impact force, which can depend on the geometry of the robot, the geometry of the environment, and the type of impact. As stated in [18], the appeal of systems with impact conditions is that short-duration effects such as high stresses, rapid dissipation of energy, and fast acceleration and deceleration may be achieved from low-energy sources. Some example current and emerging applications that motivate this field of research include manufacturing, biped walking robots, manipulation of rigid and nonrigid bodies, grasping or catching with a robotic hand, and human-machine interaction applications such as robot-assisted rehabilitation.

Largely motivated by manufacturing applications, the study of systems that undergo an impact collision has historically targeted "hard-on-hard" collisions with an infinitely large and rigid mass. As a result, the impact collision is modeled as a discontinuous event, where discontinuous controllers have been developed to compensate for the instantaneous velocity jump (e.g., [4] and [19]-[25]). For example, a discontinuous controller was designed in [24] to regulate the impact of a hydraulic actuator with a static environment. A switching controller was developed in [25] to eliminate the bouncing phenomena associated with a robot impacting a static surface. In [19], a class of switching controllers was examined for mechanical systems subject to an algebraic inequality condition and an impact rule relating the interaction impulse and the velocity. The analysis in [19] utilized a discrete Lyapunov function that required the use of the Dini derivative to examine the stability of the system. A hybrid bang-bang impedance/time-delay controller was 
developed in [20] that establishes a stable contact and achieves the desired dynamics for contact or noncontact conditions.

Although discontinuous impact models with impulsive velocity effects may effectively characterize collisions between rigid objects, these models do not capture the effects when a rigid body collides with a deformable surface. Existing models of contact with a deformable surface typically characterize the deformation as a linear or nonlinear spring with some bounded stiffness (e.g., [18] and [26]-[35]). A challenge for the development of controllers based on these models is that the stiffness may not be a priori known or may change through repeated use (e.g., strain hardening). For example, a two-degree-offreedom planar manipulator was asymptotically regulated to contact an infinitely rigid surface with a deformable second link represented by a massless linear spring in [27], where the robot controller either required measurement of the interaction force or the spring stiffness. A reduced-order observer was used in [26] to control the impact between the end-effectors of two cooperating manipulators, where the exact knowledge of the robot dynamics and stiffness of the contact was required. In [33], a backstepping approach is used to asymptotically regulate the impact of two systems where the exact system dynamics are known. In [34], a class of continuous energy-based controllers was developed that achieves global asymptotic stabilization/ regulation of an underactuated Euler-Lagrange system subject to an elastic contact with finite stiffness provided that the exact knowledge of the impact stiffness is known.

This paper considers a rigid robotic system that undergoes an impact collision with an underactuated deformable mass-spring system. The robot/mass-spring collision is modeled as a differentiable impact, as in the recent work in [18] and [34]-[36]. The dynamic model for both systems and the impact force are assumed to have uncertain parameters. This paper is specifically focused on a planar robot colliding with a mass-spring system as an academic example of a broader class of such systems. The control objective is defined as the desire to enable a robot to collide with an unactuated system and regulate the resulting coupled mass-spring robot (MSR) system to a desired compressed state despite parametric uncertainty (i.e., unknown masses and stiffness) throughout the MSR system.

Based on the aforementioned objective, one control strategy could be to implement a controller that regulated the robot to a desired constant set point associated with the desired mass-spring system. The main drawback of such an approach is that the dynamics associated with the impact collision would appear as a disturbance to the controller, requiring the controller to exploit high-gain or high-frequency feedback to ensure stability. In contrast to a regulation control strategy, the control development in this paper uses integrator backstepping [37] to design a desired robot trajectory as a virtual control input to the mass-spring system. The desired robot trajectory is designed based on a Lyapunov analysis that includes the dynamics of the impact collision and the coupled motion of the MSR system. A force control input (that can be related to the actual robot joint torque inputs) is then designed to asymptotically eliminate the mismatch between the time-varying desired robot trajectory and the actual trajectory. Unlike some other results in literature, the continuous force controller does not depend on measuring

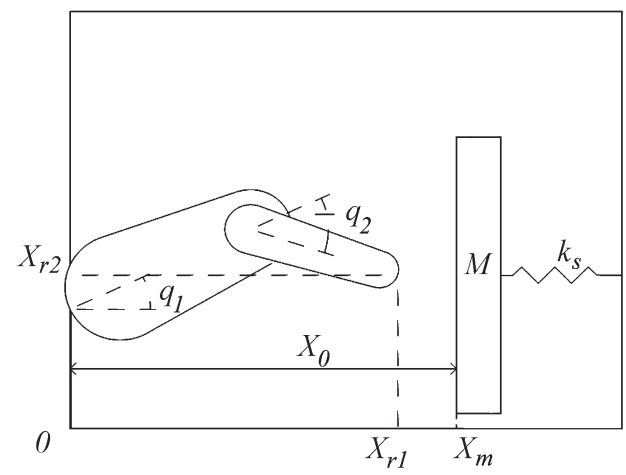

Fig. 1. MSR system is an academic example of an impact between two dynamic systems.

the impact force or the measurement of other acceleration terms and, due to the adaptive nature, does not depend on the knowledge of the masses or stiffness, although upper bounds are assumed known.

\section{Dynamic Model}

The subsequent development is motivated by the academic problem illustrated in Fig. 1. The dynamic model for the twolink revolute robot depicted in Fig. 1 can be expressed in the joint space as follows:

$$
M(q) \ddot{q}+C(q, \dot{q}) \dot{q}+h(q)=\tau
$$

where $q(t), \dot{q}(t), \ddot{q}(t) \in \mathbb{R}^{2}$ represent the angular position, the velocity, and the acceleration of the robot links, respectively, $M(q) \in \mathbb{R}^{2 \times 2}$ represents the uncertain inertia matrix, $C(q, \dot{q}) \in$ $\mathbb{R}^{2 \times 2}$ represents the uncertain centripetal Coriolis effects, $h(q) \triangleq\left[h_{1}(q), h_{2}(q)\right]^{T} \in \mathbb{R}^{2}$ represents uncertain conservative forces (e.g., gravity), and $\tau(t) \in \mathbb{R}^{2}$ represents the torque control inputs. The Euclidean position of the endpoint of the second robot link is denoted by $x_{r}(t) \triangleq\left[x_{r 1}(t), x_{r 2}(t)\right]^{T} \in \mathbb{R}^{2}$, which can be related to the joint space through the following kinematic relationship:

$$
\dot{x}_{r}=J(q) \dot{q}
$$

where $J(q) \in \mathbb{R}^{2 \times 2}$ denotes the manipulator Jacobian. The unforced dynamics of the mass-spring system in Fig. 1 is given by

$$
m \ddot{x}_{m}+k_{s}\left(x_{m}-x_{0}\right)=0
$$

where $x_{m}(t), \dot{x}_{m}(t), \ddot{x}_{m}(t) \in \mathbb{R}$ represent the displacement, the velocity, and the acceleration of the unknown mass $m \in \mathbb{R}$, $x_{0} \in \mathbb{R}$ represents the initial undisturbed position of the mass, and $k_{s} \in \mathbb{R}$ represents the unknown stiffness of the spring.

Assumption 1: We assume that $x_{r 1}(t)$ and $x_{m}(t)$ can be bounded as follows:

$$
\zeta_{x_{r}} \leq x_{r 1}(t) \quad x_{m}(t) \leq \zeta_{x_{m}}
$$

where $\zeta_{x_{r}} \in \mathbb{R}$ is a known constant that is determined by the minimum coordinate of the robot along the $X_{1}$-axis, and $\zeta_{x_{m}} \in$ $\mathbb{R}$ is a known positive constant. The lower bound assumption for $x_{r 1}(t)$ is based on the geometry of the robot, and the upper bound assumption for $x_{m}(t)$ is based on the physical fact that the mass is attached by the spring to some object, and the mass will not be able to move past that object. 
In the following, the contact model is considered as an elastic contact with finite stiffness. An impact between the second link of the robot and the spring-mass system occurs when $x_{r 1}(t) \geq$ $x_{m}(t)$. The impact will yield equal and opposite force reactions between the robot and the mass-spring system. Specifically, the impact force acting on the mass, represented by $F_{m}\left(x_{r}, x_{m}\right) \in$ $\mathbb{R}$, is assumed to have the following form [18], [36]:

$$
F_{m}=K_{I} \Lambda\left(x_{r 1}-x_{m}\right)
$$

where $K_{I} \in \mathbb{R}$ represents an unknown positive stiffness constant, and $\Lambda\left(x_{r}, x_{m}\right) \in \mathbb{R}$ is defined as

$$
\Lambda= \begin{cases}1 & x_{r 1} \geq x_{m} \\ 0 & x_{r 1}<x_{m}\end{cases}
$$

The impact force acting on the robot links produces a torque, denoted by $\tau_{d}\left(x_{r}, x_{m}, q\right) \in \mathbb{R}^{2}$, as follows:

$$
\tau_{d}=K_{I} \Lambda\left(x_{r 1}-x_{m}\right)\left[\begin{array}{c}
l_{1} \sin \left(q_{1}\right)+l_{2} \sin \left(q_{2}+q_{1}\right) \\
l_{2} \sin \left(q_{2}+q_{1}\right)
\end{array}\right]
$$

where $l_{1}, l_{2} \in \mathbb{R}$ denote the robot link lengths. Based on (1), (3), and (5)-(7), the dynamic model for the MSR system can be expressed as follows:

$$
\begin{aligned}
M(q) \ddot{q}+C(q, \dot{q}) \dot{q}+h(q)-\tau_{d} & =\tau \\
m \ddot{x}_{m}+k_{s}\left(x_{m}-x_{0}\right) & =F_{m} .
\end{aligned}
$$

After premultiplying the robot dynamics by the inverse of the Jacobian transpose and utilizing (2), the dynamics in (8) can be rewritten as

$$
\begin{aligned}
\bar{M}\left(x_{r}\right) \ddot{x}_{r}+\bar{C}\left(x_{r}, \dot{x}_{r}\right) \dot{x}_{r}+\bar{h}\left(x_{r}\right)+\left[\begin{array}{c}
F_{m} \\
0
\end{array}\right] & =F \\
m \ddot{x}_{m}+k_{s}\left(x_{m}-x_{0}\right) & =F_{m}
\end{aligned}
$$

where $F(t) \triangleq J^{-T}(q) \tau(t) \in \mathbb{R}^{2}$ denotes the manipulator force. The dynamic model in (9) exhibits the following properties that will be utilized in the subsequent analysis.

Assumption 2: During the subsequent control development, we assume that the minimum singular value of $J(q)$ is greater than a known small positive constant $\delta>0$, such that $\max \left\{\left\|J^{-1}(q)\right\|\right\}$ is known a priori, and hence, all kinematic singularities are always avoided.

Assumption 3: We assume that the mass of the mass-spring system can be upper and lower bounded as

$$
m_{l}<m<m_{u}
$$

where $m_{l}, m_{u} \in \mathbb{R}$ denote known positive bounding constants. The unknown stiffness constants $K_{I}$ and $k_{s}$ are also assumed to be bounded as follows:

$$
\underline{\zeta}_{K}<K_{I}<\bar{\zeta}_{K} \quad \underline{\zeta}_{k_{s}}<k_{s}<\bar{\zeta}_{k_{s}}
$$

where $\underline{\zeta}_{K}, \bar{\zeta}_{K}, \underline{\zeta}_{k_{s}}, \bar{\zeta}_{k_{s}} \in \mathbb{R}$ denote known positive bounding constants.

Property 1: The inertia matrix $\bar{M}\left(x_{r}\right)$ is symmetric, positive definite, and can be lower and upper bounded as follows [38], [39]:

$$
a_{1}\|\xi\|^{2} \leq \xi^{T} \bar{M} \xi \leq a_{2}\|\xi\|^{2}, \quad \forall \xi \in \mathbb{R}^{2}
$$

Property 2: The following skew-symmetric relationship is satisfied [38], [39]:

$$
\xi^{T}\left(\frac{1}{2}, \dot{\bar{M}}\left(x_{r}\right)-\bar{C}\left(x_{r}, \dot{x}_{r}\right)\right) \xi=0, \quad \forall \xi \in \mathbb{R}^{2} .
$$

Property 3: The robot dynamics given in (9) can be linearly parameterized as follows [38], [39]:

$$
Y\left(x_{r}, \dot{x}_{r}, \ddot{x}_{r}\right) \theta=\bar{M}\left(x_{r}\right) \ddot{x}_{r}+\bar{C}\left(x_{r}, \dot{x}_{r}\right) \dot{x}_{r}+\bar{h}\left(x_{r}\right)+\left[\begin{array}{c}
F_{m} \\
0
\end{array}\right]
$$

where $\theta \in \mathbb{R}^{p}$ contains the constant unknown system parameters, and $Y\left(x_{r}, \dot{x}_{r}, \ddot{x}_{r}\right) \in \mathbb{R}^{2 \times p}$ denotes the known regression matrix.

\section{Control Development}

As previously stated, the control objective is defined as the desire to enable a robot to collide with an unactuated system and regulate the resulting coupled MSR system to a desired compressed state despite parametric uncertainty (i.e., unknown masses and stiffness) throughout the MSR system. To achieve this objective, the control development is based on integrator backstepping [37] to design a desired robot trajectory as a virtual control input to the mass-spring system. The desired robot trajectory is designed based on a Lyapunov analysis that includes the dynamics of the impact collision and the coupled motion of the MSR system. A force control input (that can be related to the actual robot joint torque inputs) is then designed to asymptotically eliminate the mismatch between the time-varying desired robot trajectory and the actual trajectory. As is typical with integrator backstepping methods, the force controller depends on the time derivative of the desired robot trajectory (i.e., the virtual control input to the mass-spring system). Taking the derivative of the desired trajectory could lead to unmeasurable higher order terms (i.e., acceleration). The subsequent development exploits the hyperbolic filter structure developed in [40] and [41] to overcome the problem of injecting higher order terms in the controller and to facilitate the development of sufficient gain conditions used in the subsequent stability analysis.

\section{A. Control Objective}

The control objective is to regulate the states of an uncertain dynamic system (i.e., a two-link planar robot) that has an impact collision with another uncertain dynamic system (i.e., a mass-spring). A regulation error, denoted by $e(t) \in \mathbb{R}^{3}$, is defined to quantify this objective as

$$
e=\left[\begin{array}{ll}
e_{m} & e_{r}^{T}
\end{array}\right]^{T}
$$

where $e_{r}(t) \triangleq\left[e_{r 1}, e_{r 2}\right]^{T} \in \mathbb{R}^{2}$ and $e_{m}(t) \in \mathbb{R}$ denote the regulation error for the endpoint of the second link of the robot and mass-spring system (see Fig. 1), respectively, and are defined as follows:

$$
e_{r}=x_{r d}-x_{r} \quad e_{m}=x_{m d}-x_{m} .
$$


In (13), $x_{m d} \in \mathbb{R}$ denotes the constant known desired position of the mass, and $x_{r d}(t) \triangleq\left[x_{r d 1}(t), x_{r d 2}\right]^{T} \in \mathbb{R}^{2}$ denotes the desired position of the endpoint of the second link of the robot. The subsequent development is based on the assumption that $q(t), \dot{q}(t), x_{m}(t)$, and $\dot{x}_{m}(t)$ are measurable, and that $x_{r}(t)$ and $\dot{x}_{r}(t)$ can be obtained from $q(t)$ and $\dot{q}(t)$. To facilitate the subsequent control design and stability analysis, filtered tracking errors, ${ }^{1}$ denoted by $\eta_{m}(t) \in \mathbb{R}$ and $r_{r}(t) \in \mathbb{R}^{2}$, are defined as follows [40], [41]:

$$
\begin{aligned}
\eta_{m} & =\dot{e}_{m}+\alpha_{1} \tanh \left(e_{m}\right)+\alpha_{2} \tanh \left(e_{f}\right) \\
r_{r} & =\dot{e}_{r}+\alpha e_{r}
\end{aligned}
$$

where $\alpha, \alpha_{1}, \alpha_{2} \in \mathbb{R}$ are positive constant gains, and $e_{f}(t) \in \mathbb{R}$ is designed as follows [40], [41]:

$$
\dot{e}_{f}=-\alpha_{3} \tanh \left(e_{f}\right)+\alpha_{2} \tanh \left(e_{m}\right)-k_{1} \cosh ^{2}\left(e_{f}\right) \eta_{m}
$$

where $k_{1} \in \mathbb{R}$ is a positive constant control gain, and $\alpha_{3} \in \mathbb{R}$ is a positive constant filter gain. The filtered tracking error $r_{r}(t)$ is introduced to reduce the terms in the Lyapunov analysis [i.e., $r_{r}(t)$ can be used in lieu of including $e_{r}(t)$ and $\dot{e}_{r}(t)$ ]. The filtered tracking error $\eta_{m}(t)$ and the auxiliary signal $e_{f}(t)$ are introduced to eliminate dependence on acceleration in the subsequently designed force controller [42].

\section{B. Closed-Loop Error System}

By taking the time derivative of $m \eta_{m}(t)$ and utilizing (5), (10), (13), and (14), the following open-loop error system can be obtained:

$$
\begin{aligned}
m \dot{\eta}_{m}= & Y_{d} \theta_{d}-K_{I} \Lambda\left(x_{r 1}-x_{m}\right) \\
& +\alpha_{2} m \cosh ^{-2}\left(e_{f}\right) \dot{e}_{f}+\alpha_{1} m \cosh ^{-2}\left(e_{m}\right) \dot{e}_{m} .
\end{aligned}
$$

In (16), $Y_{d}\left(x_{m}\right)=\left(x_{m}-x_{o}\right)$ and $\theta_{d}=k_{s}$. To facilitate the subsequent analysis, the following notation is introduced [40]:

$$
\begin{aligned}
Y_{d} \theta_{d} & =Y_{d} K_{I} K_{I}^{-1} \theta_{d}=Y_{d k} \theta_{d k} \\
& =K_{I}\left(x_{m}-x_{o}\right)\left[\begin{array}{c}
k_{s} \\
K_{I}
\end{array}\right] .
\end{aligned}
$$

After using (14) and (15), the expression in (16) can be rewritten as follows:

$$
\begin{aligned}
m \dot{\eta}_{m}=Y_{d} \theta_{d}+ & K_{I}\left(x_{r d 1}-\Lambda x_{r 1}\right) \\
& +K_{I} \Lambda x_{m}-K_{I} x_{r d 1}+\chi-\alpha_{2} m k_{1} \eta_{m}
\end{aligned}
$$

where $\chi\left(e_{m}, e_{f}, \eta_{m}, t\right) \in \mathbb{R}$ is an auxiliary term defined as

$$
\begin{aligned}
\chi= & \alpha_{1} m \cosh ^{-2}\left(e_{m}\right)\left(\eta_{m}-\alpha_{1} \tanh \left(e_{m}\right)\right) \\
& -\alpha_{1} \alpha_{2} m \cosh ^{-2}\left(e_{m}\right) \tanh \left(e_{f}\right) \\
& +\alpha_{2} m \cosh ^{-2}\left(e_{f}\right)\left(-\alpha_{3} \tanh \left(e_{f}\right)\right) \\
& +\alpha_{2} m \cosh ^{-2}\left(e_{f}\right)\left(\alpha_{2} \tanh \left(e_{m}\right)\right) .
\end{aligned}
$$

The motivation for the introduction of the filter signals $\eta_{m}(t)$ and $e_{f}(t)$ and the selective grouping of the terms in (19) allow

\footnotetext{
${ }^{1}$ The term filtered tracking error is used to indicate that the filter input [e.g., $\left.e_{r}(t)\right]$ is equal to a low-pass filtered version of the output [e.g., $r_{r}(t)$ ].
}

the development of the following linear inequality (versus a quadratic inequality):

$$
|\chi| \leq \zeta_{1}\|z\|
$$

where $\zeta_{1} \in \mathbb{R}$ is a positive bounding constant, and $z(t) \in \mathbb{R}^{3}$ is defined as follows:

$$
z=\left[\begin{array}{ll}
\eta_{m} & \tanh \left(e_{m}\right) \quad \tanh \left(e_{f}\right)
\end{array}\right]
$$

That is, the use of hyperbolic functions in the development of $\eta_{m}(t)$ and $e_{f}(t)$ allows the linear inequality in (20) to be developed; without the hyperbolic functions, the bound would be quadratic. Feedback terms in the controller can be used to damp out terms that are bounded by a linear function of the states without restricting the domain of the stability result as demonstrated in the subsequent stability analysis. If the hyperbolic terms had not been used in the filter structure, the bound in (20) would have been quadratic, potentially limiting the domain of the stability result (i.e., a semiglobal result).

Based on (18) and the subsequent stability analysis, the desired robot link position is designed as follows:

$$
\begin{aligned}
x_{r d 1}= & Y_{d} \hat{\theta}_{d k}+x_{m}+k_{2} \tanh \left(e_{m}\right) \\
& -k_{1} k_{2} \cosh ^{2}\left(e_{f}\right) \tanh \left(e_{f}\right) \\
x_{r d 2}= & \varepsilon .
\end{aligned}
$$

In (22), $\varepsilon \in \mathbb{R}$ is an appropriate positive constant (i.e., $\varepsilon$ is selected, so the robot will impact the mass-spring system in the vertical direction), $k_{2} \in \mathbb{R}$ is a positive constant control gain, and the control gain $k_{1} \in \mathbb{R}$ is defined as

$$
k_{1}=\frac{1}{m_{l}}\left(3+k_{n 1} \zeta_{1}^{2}\right)
$$

where $k_{n 1} \in \mathbb{R}$ is a positive constant nonlinear damping gain. The parameter estimate $\hat{\theta}_{d k}(t) \in \mathbb{R}$ in (22) is generated by the adaptive update law, i.e.,

$$
\dot{\hat{\theta}}_{d k}=\operatorname{proj}\left(\Gamma Y_{d} \eta_{m}\right) .
$$

In (24), $\Gamma \in \mathbb{R}$ is a positive constant, and $\operatorname{proj}(\cdot)$ denotes a sufficiently smooth projection algorithm [43] utilized to guarantee that $\hat{\theta}_{d k}(t)$ can be bounded as follows:

$$
\underline{\theta}_{d k} \leq \hat{\theta}_{d k} \leq \bar{\theta}_{d k}
$$

where $\underline{\theta}_{d k}, \bar{\theta}_{d k} \in \mathbb{R}$ denote known constant lower and upper bounds for $\theta_{d k}(t)$, respectively.

After substituting (22) into (18), the closed-loop error system for $\eta_{m}(t)$ can be obtained as follows:

$$
\begin{aligned}
m \dot{\eta}_{m}= & K_{I}\left(x_{r d 1}-\Lambda x_{r 1}\right)+K_{I}\left(\Lambda x_{m}-x_{m}\right) \\
& +K_{I} k_{1} k_{2} \cosh ^{2}\left(e_{f}\right) \tanh \left(e_{f}\right)+Y_{d k} \tilde{\theta}_{d k} \\
& -K_{I} k_{2} \tanh \left(e_{m}\right)+\chi-\alpha_{2} m k_{1} \eta_{m}
\end{aligned}
$$

In (26), the parameter estimation error $\tilde{\theta}_{d k}(t) \in \mathbb{R}$ is defined as

$$
\tilde{\theta}_{d k}=\theta_{d k}-\hat{\theta}_{d k}
$$

The open-loop robot error system can be obtained by taking the time derivative of $r_{r}(t)$ and premultiplying by the robot 
inertia matrix as follows:

$$
\bar{M} \dot{r}_{r}=Y_{r} \theta_{r}-\bar{C} r_{r}-F
$$

where (9), (13), and (14) were utilized, and

$$
\begin{aligned}
Y_{r} \theta_{r}=\bar{M} \ddot{x}_{r d}+\alpha \bar{M} \dot{e}_{r}+\bar{h}+\bar{C} \dot{x}_{r d} \\
+\alpha \bar{C} x_{r d}+\left[\begin{array}{c}
K_{I} \Lambda\left(x_{r 1}-x_{m}\right) \\
0
\end{array}\right]-\alpha \bar{C} x_{r}
\end{aligned}
$$

where $Y_{r}\left(x_{r}, \dot{x}_{r}, x_{m}, \dot{x}_{m}, e_{f}, \eta_{m}, t\right) \in \mathbb{R}^{2 \times P}$ denotes a known regression matrix, and $\theta_{r} \in \mathbb{R}^{P}$ denotes an unknown constant parameter vector. See Appendix A for a linearly parameterizable expression for $\bar{M}\left(x_{r}\right) \ddot{x}_{r d}(t)$ that does not depend on acceleration terms. Based on (27) and the subsequent stability analysis, the robot force control input is designed as follows:

$$
F=Y_{r} \hat{\theta}_{r}+e_{r}+k_{3} r_{r}
$$

where $k_{3} \in \mathbb{R}$ is a positive constant control gain, and $\hat{\theta}_{r}(t) \in$ $\mathbb{R}^{P}$ is an estimate for $\theta_{r}$ generated by the following adaptive update law:

$$
\dot{\hat{\theta}}_{r}=\operatorname{proj}\left(\Gamma_{r} Y_{r}^{T} r_{r}\right) .
$$

In (30), $\Gamma_{r} \in \mathbb{R}^{P \times P}$ is a positive-definite constant diagonal adaptation gain matrix, and $\operatorname{proj}(\cdot)$ denotes a projection algorithm utilized to guarantee that the $i$ th element of $\hat{\theta}_{r}(t)$ can be bounded as follows:

$$
\underline{\theta}_{r i} \leq \hat{\theta}_{r i} \leq \bar{\theta}_{r i}
$$

where $\underline{\theta}_{r i}, \bar{\theta}_{r i} \in \mathbb{R}$ denote known constant lower and upper bounds for each element of $\theta_{r}(t)$, respectively.

The closed-loop error system for $r_{r}(t)$ can be obtained after substituting (29) into (27) as follows:

$$
\bar{M} \dot{r}_{r}=Y_{r} \tilde{\theta}_{r}-k_{3} r_{r}-\bar{C} r_{r}-e_{r} .
$$

In (31), the parameter estimation error $\tilde{\theta}_{r}(t) \in \mathbb{R}^{P}$ is defined as

$$
\tilde{\theta}_{r}=\theta_{r}-\hat{\theta}_{r}
$$

Remark 1: Based on (29), the control torque input can be expressed as follows:

$$
\tau=J^{T}\left(Y_{r} \hat{\theta}_{r}+e_{r}+k_{3} r_{r}\right)
$$

where $J(q)$ denotes the manipulator Jacobian introduced in (2).

\section{STABILITY ANALYSIS}

Theorem: The controller given by (22), (24), (29), and (30) ensures asymptotic regulation of the MSR system in the sense that the mass-spring system converges to the desired set point, and the robot links converge to the desired trajectory as follows:

$$
\left|e_{m}(t)\right| \rightarrow 0 \quad\left\|e_{r}(t)\right\| \rightarrow 0, \quad \text { as } t \rightarrow \infty
$$

provided that $k_{1}, k_{2}$, and $k_{n 1}$ are selected sufficiently large (see Appendix B), and the following sufficient gain condition is satisfied:

$$
\alpha_{2}>\max \left\{\frac{1}{\alpha},\left(\zeta_{x_{m}}+\left|\zeta_{x_{r}}\right|\right)^{2}\right\} \frac{\bar{\zeta}_{K}^{2}}{4}
$$

where $\zeta_{x_{m}}, \zeta_{x_{r}}, \bar{\zeta}_{K}$, and $\alpha$ are defined in (4), (11), and (14), respectively.

Proof: See Appendix B.

Remark 2: The sufficient gain condition in (34) indicates that as $K_{I}$ becomes infinitely large, $\alpha_{2}$ must also grow infinitely large. See the classic discussion on this issue given in [44]. In this result, we only consider contact with surfaces with finite $K_{I}$. In the experimental results for this paper, the actual values for $\alpha_{2}$ were selected much lower than the sufficient condition in (34) indicates, as is typical in nonlinear control designs.

Remark 3: As is typical in the literature, the controller developed in (22), (24), (29), and (30) is based on the underlying assumption that arbitrarily large (but finite) control authority is available. A potential disadvantage of the controller is that the gain conditions developed in Appendix B and in (34) indicate that $k_{n 1}, k_{2}$, and $\alpha_{2}$, respectively, should be selected sufficiently large. As demonstrated by the subsequent experimental results, the gains may be selected much lower in practice (i.e., the gain conditions are the result of a conservative Lyapunov analysis). However, the subsequent experimental section also illustrates that even when the gain conditions are violated, large initial conditions and a high stiffness coefficient result in a highgain controller that initially saturates the actuators. The control torque in the experiment was artificially saturated to reduce the magnitude of the impact to protect a capacitance probe from contact by excessive bending of the aluminum rod/spring assembly. Research that can limit the required control torque for systems that undergo an impact collision seems to be an interesting open problem.

\section{EXPERIMENTAL RESULTS}

The results in this section are not intended to represent the best possible results that can be obtained by the controller. Different control gains and initial conditions will yield different results. The motivation for the experimental results in this section is to demonstrate the capability of the controller to yield a certain level of performance (see Figs. 4-12).

The test bed depicted in Figs. 2 and 3 was developed for experimental demonstration of the proposed controller. The test bed is composed of a mass-spring system and a twolink robot. The body of the mass-spring system includes a U-shaped aluminum plate [item (8) in Fig. 2] mounted on an undercarriage with porous carbon air bearings, which enables the undercarriage to glide on an air cushion over a glass-covered aluminum rail. A steel core spring [item (1) in Fig. 2] connects the undercarriage to an aluminum frame, and a linear variable displacement transducer [LVDT; item (2) in Fig. 2] is used to measure the position of the undercarriage assembly. The impact surface consists of an aluminum plate connected to the undercarriage assembly through a stiff spring mechanism [item (7) in Fig. 2]. A capacitance probe [item (3) in Fig. 2] is used to measure the deflection of the stiff spring mechanism. The two-link robot [items (4-6) in Fig. 2] is made of two aluminum 


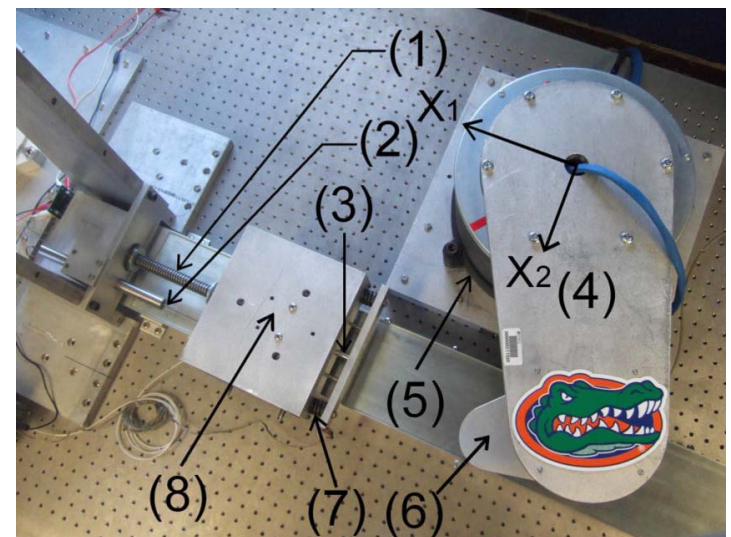

Fig. 2. Top view of the experimental test bed, including (1) spring, (2) LVDT, (3) capacitance probe, (4) link1, (5) motor1, (6) link2, (7) stiff spring mechanism, and (8) mass.

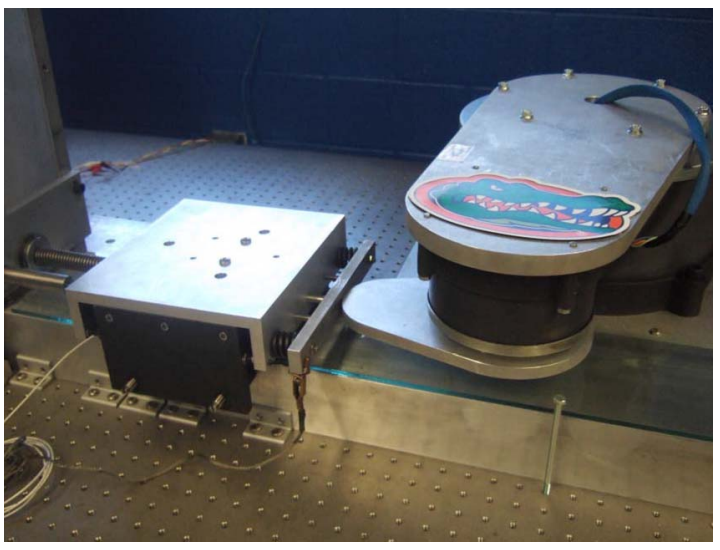

Fig. 3. Side view of the experimental test bed.

links, mounted on $240.0 \mathrm{~N} \cdot \mathrm{m}$ (base link) and $20.0 \mathrm{~N} \cdot \mathrm{m}(\mathrm{sec}-$ ond link) direct-drive switched reluctance motors. The motors are controlled through power electronics operating in the torque control mode. The motor resolvers provide rotor position measurements with a resolution of 614400 pulses/revolution, and a standard backward difference algorithm is used to numerically determine the velocity from the encoder readings. A Pentium 2.8-GHz PC operating under QNX hosts the control algorithm, which was implemented via a custom graphical user interface [45] to facilitate real-time graphing, data logging, and the ability to adjust control gains without recompiling the program. Data acquisition and control implementation were performed at a frequency of $2.0 \mathrm{kHz}$ using the ServoToGo I/O board.

The control gains $\alpha$ and $k_{3}$, defined as scalars in (14) and (29), were implemented (with nonconsequential implications to the stability result) as diagonal gain matrices to provide more flexibility in the experiment. Specifically, the control gains were selected as follows:

$$
\begin{aligned}
k_{1} & =0.18 \\
k_{2} & =0.9 \\
k_{3} & =\operatorname{diag}\{185,170\} \\
\alpha_{1} & =45 \\
\alpha_{2} & =8 \\
\alpha_{3} & =0.01 \\
\alpha & =\operatorname{diag}\{60,90\} .
\end{aligned}
$$

The control gains in (35) were obtained by choosing gains and then adjusting based on performance. If the response exhibited a prolonged transient response (compared with the response obtained with other gains), the proportional gains were adjusted. If the response exhibited overshoot, derivative gains were adjusted. Last, to fine-tune the performance, the adaptive gains were adjusted after the feedback gains were tuned as described to yield the best performance. As a result of a conservative stability analysis, the final gains used may not satisfy the sufficient gain conditions developed in the theorem proof provided in Appendix B. The subsequent results indicate that the developed controller can be applied despite the fact that some gain conditions are not satisfied. In contrast to the above approach, the control gains could potentially have been adjusted using more methodical approaches. For example, the nonlinear system in [46] was linearized at several operating points, and a linear controller was designed for each point; moreover, the gains were chosen by interpolating or scheduling the linear controllers. In [47], a neural network is used to tune the gains of a PID controller. In [48], a genetic algorithm was used to finetune the gains after an initial guess was made by the controller designer. Additionally, in [49], the tuning of a PID controller for robot manipulators is discussed.

The adaptation gains were selected as follows:

$$
\begin{aligned}
\Gamma & =90 \\
\Gamma_{r} & =\operatorname{diag}\left\{\begin{array}{l}
4.01 \times 10^{12}, 1.2 \times 10^{7}, 0.2,3.3 \times 10^{12}, 6 \times 10^{6}, \\
\left.0.1,2.4 \times 10^{11}, 7 \times 10^{5}, 0.1,2.35 \times 10^{11}\right\} .
\end{array}\right.
\end{aligned}
$$

The adaptation gains $\Gamma_{r}$ in (36) are used to enable the adaptive estimate to sufficiently change relative to the large values of the uncertain parameters in $\theta_{r}$. Smaller adaptation gains could be used to obtain different results. The initial conditions for the robot coordinates and the mass-spring position were given by (in meters)

$$
\left[\begin{array}{lll}
x_{r 1}(0) & x_{r 2}(0) & x_{m}(0)
\end{array}\right]=\left[\begin{array}{lll}
0.008 & 0.481 & 0.202
\end{array}\right] .
$$

The initial velocities of the robot and the mass-spring were zero, and the desired mass-spring position was given by (in meters)

$$
x_{m d}=0.232 \text {. }
$$

That is, the tip of the second link of the robot was initially $224 \mathrm{~mm}$ from the desired set point and $194 \mathrm{~mm}$ from $x_{0}$ along the $X_{1}$-axis (see Fig. 2). Once the initial impact occurs, the robot is required to depress the spring [item (1) in Fig. 2] to move the mass $30 \mathrm{~mm}$ along the $X_{1}$-axis.

The mass-spring and robot errors [i.e., $e(t)]$ are shown in Figs. 4 and 5. The peak steady-state position errors of the endpoint of the second link of the robot along the $X_{1}$-axis [i.e., $\left.\left|e_{r 1}(t)\right|\right]$ and along the $X_{2}$-axis [i.e., $\left.\left|e_{r 2}(t)\right|\right]$ are $0.212 \mathrm{~mm}$ and $5.77 \mu \mathrm{m}$, respectively. The peak steady-state position error of the mass [i.e., $\left|e_{m}(t)\right|$ ] is $2.72 \mu \mathrm{m}$. The $0.212-\mathrm{mm}$ maximum steady-state error in $\left|e_{r 1}(t)\right|$ is due to the $Y_{d} \hat{\theta}_{d k}(t)$ term of $x_{r d 1}(t)$ in (22), where $Y_{d}\left(x_{m}\right)$ is approximately $0.03 \mathrm{~m}$, and $\hat{\theta}_{d k}(t)$ has a maximum steady-state value of $0.007(\mathrm{~N} / \mathrm{m}) /$ 
(a)

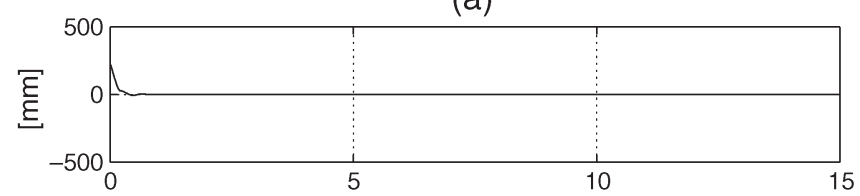

(b)

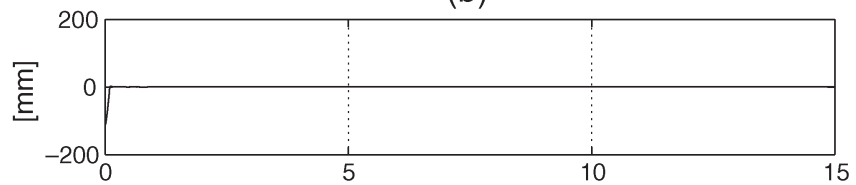

(c)

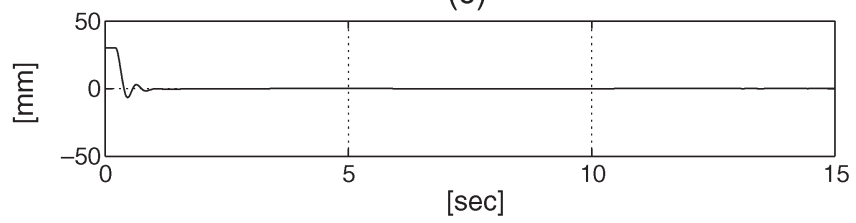

Fig. 4. Mass-spring and robot errors $e(t)$. (a) Position error of the robot tip along the $X_{1}$-axis [i.e., $\left.e_{r 1}(t)\right]$. (b) Position error of the robot tip along the $X_{2}$-axis [i.e., $\left.e_{r 2}(t)\right]$. (c) Position error of the mass-spring [i.e., $e_{m}(t)$ ].

(a)

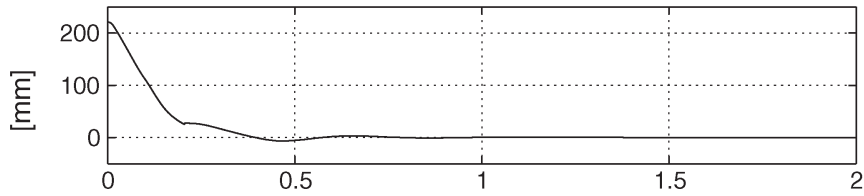

(b)

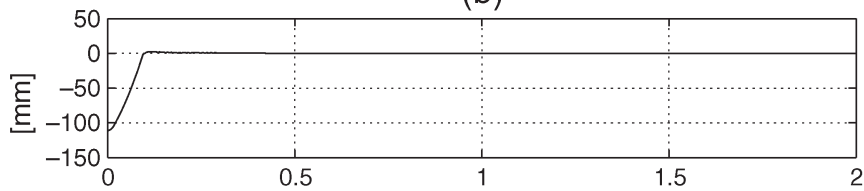

(c)

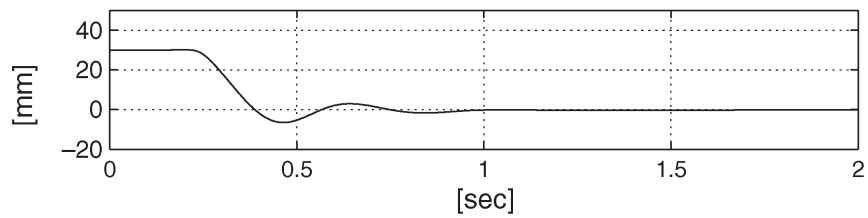

Fig. 5. Mass-spring and robot errors $e(t)$ during the initial $2 \mathrm{~s}$.

$(\mathrm{N} / \mathrm{m})$, yielding a 0.21 -mm error. All of the other terms in $e_{r 1}(t)$ are negligible at the steady state.

The input control torques in (33) are shown in Figs. 6 and 7. To constrain the impact force to a level that ensured that the aluminum plate did not flex to the point of contact with the capacitance probe, the computed torques are artificially saturated. Fig. 6 depicts the computed torques, and Fig. 7 depicts the actual torques (solid line) along with the computed torques (dashed line). The resulting desired trajectory along the $X_{1}$-axis [i.e., $\left.x_{r d 1}(t)\right]$ is depicted in Fig. 8, and the desired trajectory along the $X_{2}$-axis was chosen as $x_{r d 2}=370 \mathrm{~mm}$. Fig. 9 depicts the value of $\hat{\theta}_{d k}(t) \in \mathbb{R}$, and Figs. 10-12 depict the values of $\hat{\theta}_{r}(t) \in \mathbb{R}^{10}$. The order of the curves in the plots is based on the relative scale of the parameter estimates rather than the numerical order in $\hat{\theta}_{r}(t)$. A video of the experiment is provided in [50]. (a)

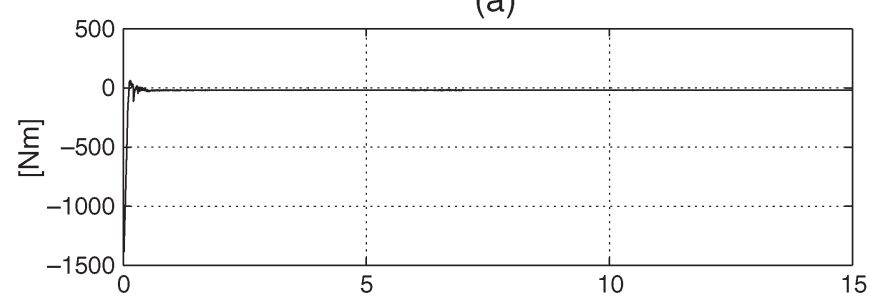

(b)

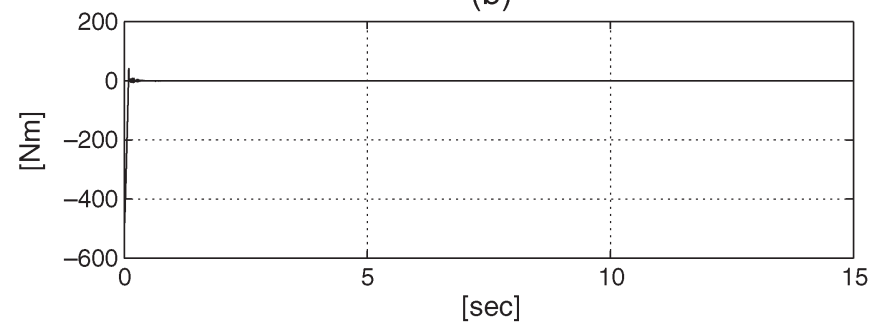

Fig. 6. Computed control torques $J^{T}(q) F(t)$ for (a) base motor and (b) second-link motor.

(a)

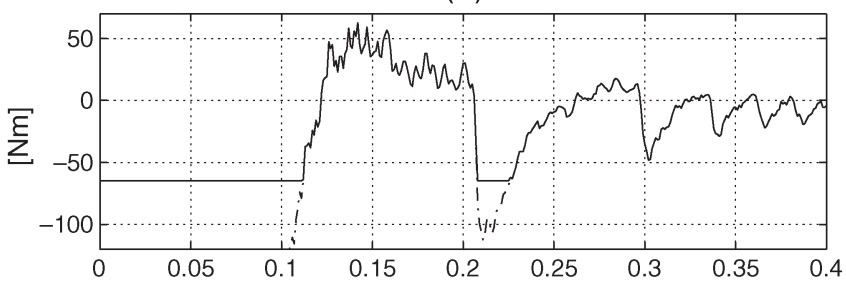

(b)

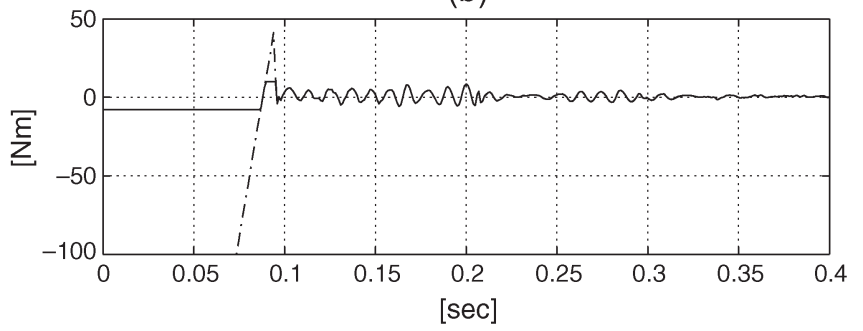

Fig. 7. Applied control torques $J^{T}(q) F(t)$ (solid line) versus computed control torques (dashed line) for (a) base motor and (b) second-link motor.

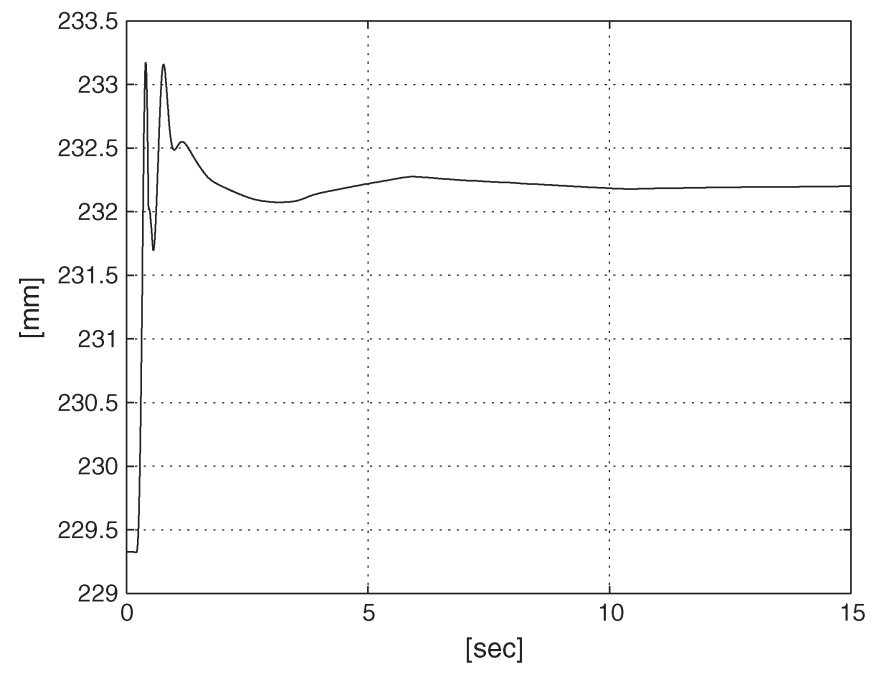

Fig. 8. Computed desired robot trajectory, $x_{r d 1}(t)$. 


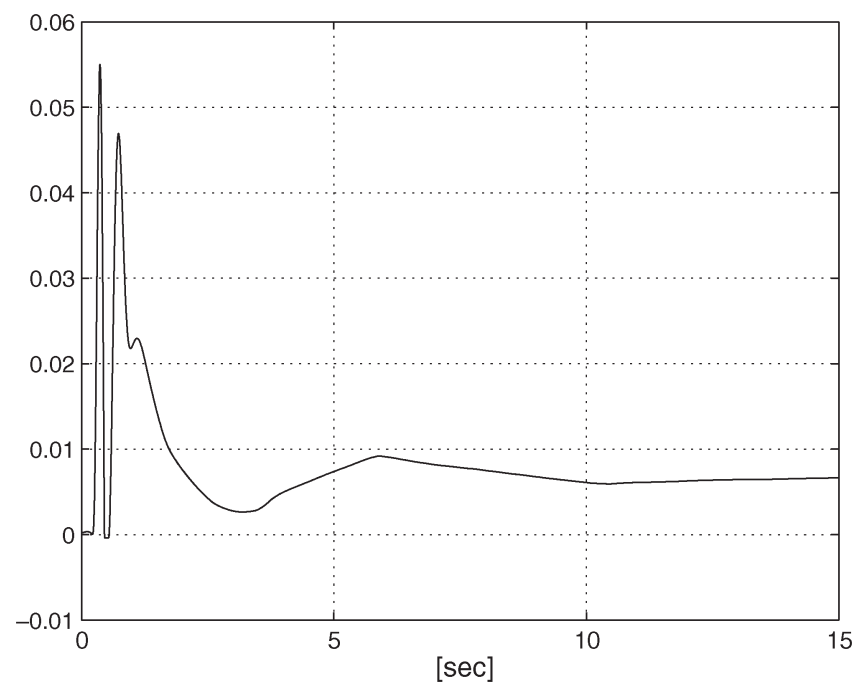

Fig. 9. Unitless parameter estimate $\hat{\theta}_{d k}(t)$ introduced in (22).

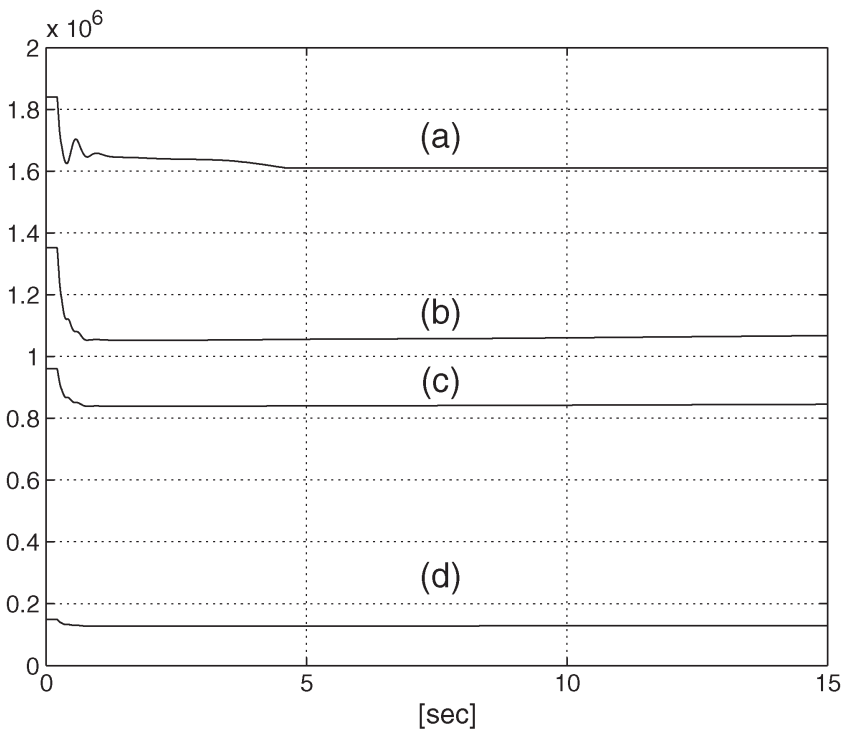

Fig. 10. Estimate for the unknown constant parameter vector $\hat{\theta}_{r}(t)$. (a) $\hat{\theta}_{r 10}(t)=K_{I}$, (b) $\hat{\theta}_{r 4}(t)=K_{I} m_{s} / m$, (c) $\hat{\theta}_{r 1}(t)=K_{I} m_{1} / m$, and (d) $\hat{\theta}_{r 7}(t)=K_{I} m_{2} / m$, where $m_{1}, m_{2} \in \mathbb{R}$ denotes the mass of the first and second links of the robot, respectively, $m_{s} \in \mathbb{R}$ denotes the mass of the motor connected to the second link of the robot, and $m \in \mathbb{R}$ denotes the mass of the mass-spring system.

\section{CONCLUSION}

An adaptive nonlinear controller is proven to regulate the states of a planar robot colliding with an unactuated mass-spring system. The continuous controller yields asymptotic regulation of the spring-mass and robot links. New control design, error system development, and stability analysis techniques were required to compensate for the fact that the dynamics changed from an uncoupled state to a coupled state. Experimental results are provided to illustrate the successful performance of the controller. Sufficient conditions developed in the stability analysis indicate that the control gains should be selected large enough to minimize the closed-loop steadystate error; however, high gains could result in large torques for large initial errors. The high-gain problem is exacerbated in the developed result because of the presence of the estimated

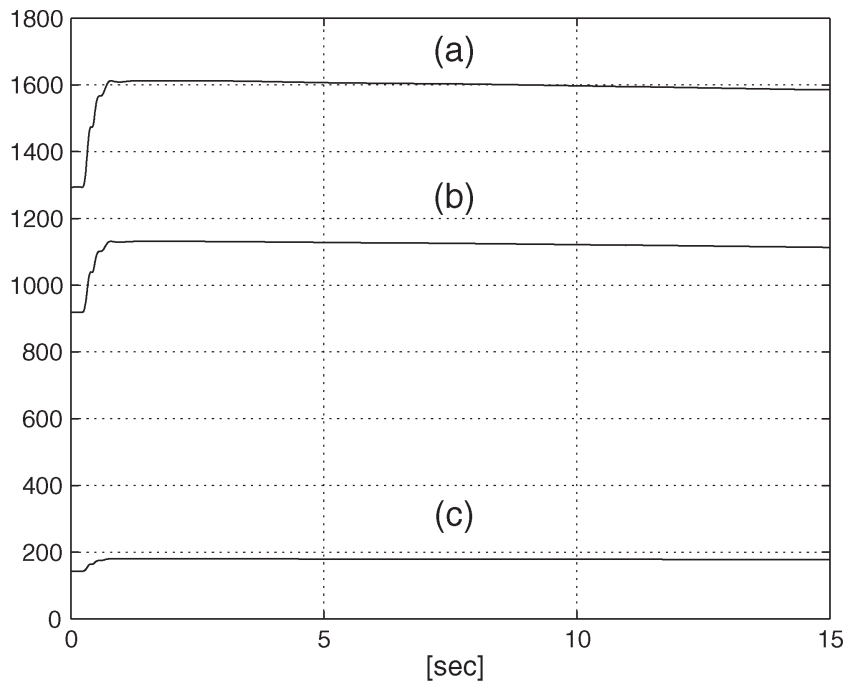

Fig. 11. Estimate for the unknown constant parameter vector $\hat{\theta}_{r}(t)$. (a) $\hat{\theta}_{r 5}(t)=k_{s} m_{s} / m$. (b) $\hat{\theta}_{r 2}(t)=k_{s} m_{1} / m$. (c) $\hat{\theta}_{r 8}(t)=k_{s} m_{2} / m$.

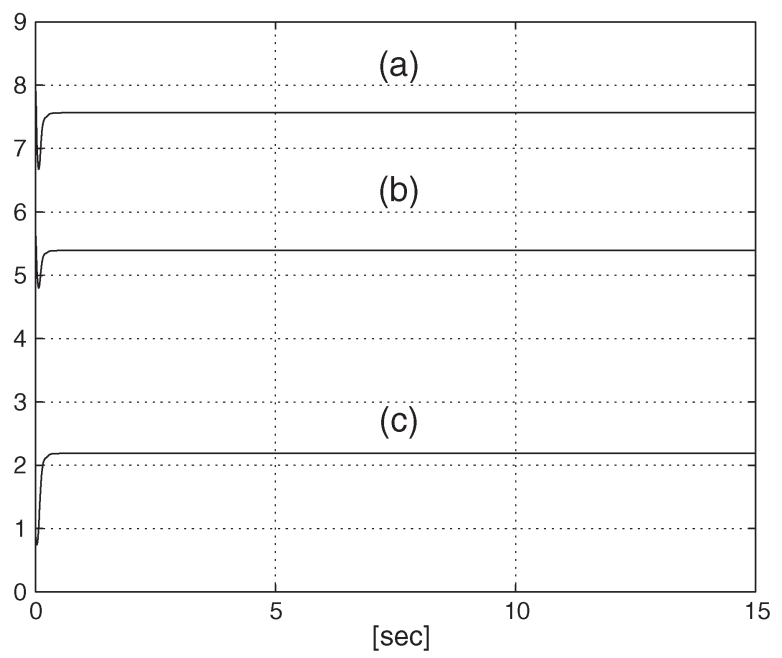

Fig. 12. Estimate for the unknown constant parameter vector $\hat{\theta}_{r}(t)$. (a) $\hat{\theta}_{r 6}(t)=m_{s}$. (b) $\hat{\theta}_{r 3}(t)=m_{1}$. (c) $\hat{\theta}_{r 9}(t)=m_{2}$.

impact stiffness coefficient. The experimental results were obtained by artificially saturating the torque to prevent damage to the capacitance probe. These issues point to a need to develop controllers that account for limited actuation.

\section{APPENDIX A \\ EXPRESSION FOR $\ddot{x}_{r d}(t)$}

Since $x_{r d 2}$ is a constant, the subsequent development is only focused on determining $\ddot{x}_{r d 1}(t)$. After using (13), (15), (22), and (24), the first time derivative of $x_{r d 1}(t)$ can be determined as follows:

$$
\begin{aligned}
\dot{x}_{r d 1}= & Y_{d}\left(\operatorname{proj}\left(\Gamma Y_{d} \eta_{m}\right)\right)+\left(\hat{\theta}_{d k}+1-k_{2} \cosh ^{-2}\left(e_{m}\right)\right) \dot{x}_{m} \\
& -k_{1} k_{2}\left(\sinh ^{2}\left(e_{f}\right)+\cosh ^{2}\left(e_{f}\right)\right) \\
& \times\left(-\alpha_{3} \tanh \left(e_{f}\right)+\alpha_{2} \tanh \left(e_{m}\right)-k_{1} \cosh ^{2}\left(e_{f}\right) \eta_{m}\right) .
\end{aligned}
$$


Based on the fact that the projection algorithm for $\dot{\hat{\theta}}_{d k}(t)$ is designed to be sufficiently smooth [43], the expressions in (24) and (37) can be used to determine the second time derivative of $x_{r d 1}(t)$ as follows:

$$
\begin{aligned}
\ddot{x}_{r d 1}= & Y_{d} \frac{\partial\left(\operatorname{proj}\left(\Gamma Y_{d} \eta_{m}\right)\right)}{\partial \eta_{m}} \dot{\eta}_{m} \\
& +\left(Y_{d} \frac{\partial\left(\operatorname{proj}\left(\Gamma Y_{d} \eta_{m}\right)\right)}{\partial x_{m}}+2 \operatorname{proj}\left(\Gamma Y_{d} \eta_{m}\right)\right) \dot{x}_{m} \\
& -2 k_{2} \cosh ^{-3}\left(e_{m}\right) \sinh \left(e_{m}\right) \dot{x}_{m}^{2} \\
& +\left(\hat{\theta}_{d k}+1-k_{2} \cosh ^{-2}\left(e_{m}\right)\right) \ddot{x}_{m} \\
& -4 k_{1} k_{2}\left(\sinh ^{2}\left(e_{f}\right) \cosh \left(e_{f}\right)\right) \dot{e}_{f}^{2} \\
& -k_{1} k_{2}\left(\sinh ^{2}\left(e_{f}\right)+\cosh ^{2}\left(e_{f}\right)\right) \\
& \times\left(-\alpha_{3} \cosh ^{-2}\left(e_{f}\right)-2 k_{1} \cosh \left(e_{f}\right) \sinh \left(e_{f}\right) \eta_{m}\right) \dot{e}_{f} \\
& +k_{1} k_{2}\left(\sinh ^{2}\left(e_{f}\right)+\cosh ^{2}\left(e_{f}\right)\right) \\
& \times\left(-\alpha_{2} \cosh ^{-2}\left(e_{m}\right) \dot{e}_{m}+k_{1} \cosh ^{2}\left(e_{f}\right) \dot{\eta}_{m}\right) .
\end{aligned}
$$

After substituting (15) and (16) into (38) for $\dot{e}_{f}(t)$ and $\dot{\eta}_{m}(t)$, respectively, and substituting (5) and (8) into (38) for $\ddot{x}_{m}(t)$, the expression for $\bar{M}\left(x_{r}\right) \ddot{x}_{r d}(t)$ in the linear parameterization in (28) can be determined without requiring acceleration measurements.

\section{APPENDIX B \\ THEOREM PROOF}

In the following proof, a Lyapunov function and its derivative are provided. The analysis is then separated into two cases-contact and noncontact. For the noncontact case, the stability analysis indicates that the controller and error signals are bounded and converge to an arbitrarily small region. Additional analysis indicates that within this region, contact must occur. When contact occurs, a Lyapunov analysis is provided that illustrates that the MSR system asymptotically converges to the desired set point.

Proof: Let $V\left(r_{r}, e_{r}, e_{m}, e_{f}, \eta_{m}, \tilde{\theta}_{r}, \tilde{\theta}_{d k}, t\right) \in \mathbb{R}$ denote the following nonnegative radially unbounded function (i.e., a Lyapunov function candidate):

$$
\begin{aligned}
V= & \frac{1}{2} r_{r}^{T} \bar{M} r_{r}+\frac{1}{2} \tilde{\theta}_{r}^{T} \Gamma_{r}^{-1} \tilde{\theta}_{r}+\frac{1}{2} \tilde{\theta}_{d k}^{T} K_{I} \Gamma^{-1} \tilde{\theta}_{d k}+k_{2} K_{I} \\
& \times\left[\ln \left(\cosh \left(e_{m}\right)\right)+\ln \left(\cosh \left(e_{f}\right)\right)\right]+\frac{1}{2} e_{r}^{T} e_{r}+\frac{1}{2} m \eta_{m}^{2} .
\end{aligned}
$$

The time derivative of (39) can be determined as follows:

$$
\begin{aligned}
\dot{V}= & r_{r}^{T} \bar{M} \dot{r}_{r}+\frac{1}{2} r_{r}^{T} \dot{\bar{M}} r_{r}+\tilde{\theta}_{r}^{T} \Gamma_{r}^{-1} \dot{\tilde{\theta}}_{r} \\
& +k_{2} K_{I}\left[\tanh \left(e_{m}\right) \dot{e}_{m}+\tanh \left(e_{f}\right) \dot{e}_{f}\right] \\
& +\tilde{\theta}_{d k}^{T} K_{I} \Gamma^{-1} \dot{\tilde{\theta}}_{d k}+e_{r}^{T} \dot{e}_{r}+\eta_{m} m \dot{\eta}_{m} .
\end{aligned}
$$

After using (12), (14), (15), (23), (24), (26), and (30)-(32), the expression in (40) can be rewritten as

$$
\begin{aligned}
\dot{V} \leq & -k_{3} r_{r}^{T} r_{r}-\alpha_{1} k_{2} K_{I} \tanh ^{2}\left(e_{m}\right)-3 \alpha_{2} \eta_{m}^{2} \\
& -k_{2} K_{I} \alpha_{3} \tanh ^{2}\left(e_{f}\right)-k_{n 1} \zeta_{1}^{2} \alpha_{2} \eta_{m}^{2}-\alpha e_{r}^{T} e_{r}+\eta_{m} \\
& \times\left[K_{I}\left(x_{r d 1}-\Lambda x_{r 1}\right)+K_{I}\left(\Lambda x_{m}-x_{m}\right)+\chi\right] .
\end{aligned}
$$

The expression in (41) will now be examined under two different scenarios.

\section{Case 1: Noncontact}

For this case, the systems are not in contact $(\Lambda=0)$, and (41) can be rewritten as follows:

$$
\begin{aligned}
\dot{V} \leq & -k_{3} r_{r}^{T} r_{r}-\alpha_{1} k_{2} K_{I} \tanh ^{2}\left(e_{m}\right)-k_{2} K_{I} \alpha_{3} \tanh ^{2}\left(e_{f}\right) \\
& -3 \alpha_{2} \eta_{m}^{2}-k_{n 1} \zeta_{1}^{2} \alpha_{2} \eta_{m}^{2}-\alpha e_{r}^{T} e_{r} \\
& +\eta_{m}\left[K_{I} x_{r d 1}-K_{I} x_{m}+\chi\right] .
\end{aligned}
$$

Rewriting $x_{r d 1}(t)$ and substituting for $\chi\left(e_{m}, e_{f}, \eta_{m}, t\right)$ yield

$$
\begin{aligned}
\dot{V} \leq & -k_{3} r_{r}^{T} r_{r}-\alpha_{1} k_{2} K_{I} \tanh ^{2}\left(e_{m}\right)-k_{2} K_{I} \alpha_{3} \tanh ^{2}\left(e_{f}\right) \\
& -2 \alpha_{2} \eta_{m}^{2}-\left[\alpha\left\|e_{r}\right\|^{2}-\bar{\zeta}_{K}\left|\eta_{m}\right|\left\|e_{r}\right\|\right] \\
& -\left[k_{n 1} \alpha_{2} \zeta_{1}^{2} \eta_{m}^{2}-\zeta_{1}\|z\|\left|\eta_{m}\right|\right] \\
& -\left[\alpha_{2} \eta_{m}^{2}-\bar{\zeta}_{K}\left|\eta_{m}\right|\left|x_{m}-x_{r 1}\right|\right] .
\end{aligned}
$$

Completing the squares on the bracketed terms yields

$$
\begin{aligned}
\dot{V} \leq & -k_{3} r_{r}^{T} r_{r}-\alpha_{1} k_{2} K_{I} \tanh ^{2}\left(e_{m}\right)-k_{2} K_{I} \alpha_{3} \tanh ^{2}\left(e_{f}\right) \\
& -2 \alpha_{2} \eta_{m}^{2}-\left[\alpha\left(\left\|e_{r}\right\|+\frac{\bar{\zeta}_{K}\left|\eta_{m}\right|}{2}\right)^{2}\right]+\frac{\bar{\zeta}_{K}^{2}\left|\eta_{m}\right|^{2}}{4 \alpha} \\
& -\left[k_{n 1} \alpha_{2} \zeta_{1}^{2}\left(\eta_{m}+\frac{\|z\|}{2 k_{n 1} \alpha_{2} \zeta_{1}}\right)^{2}\right]+\frac{\|z\|^{2}}{4 \alpha k_{n 1}} \\
& -\left[\alpha_{2}\left(\eta_{m}+\frac{\bar{\zeta}_{K}\left|x_{m}-x_{r 1}\right|}{2 \alpha_{2}}\right)^{2}\right]+\frac{\bar{\zeta}_{K}^{2}\left|x_{m}-x_{r 1}\right|^{2}}{4 \alpha_{2}} .
\end{aligned}
$$

After upper bounding $\dot{V}(t)$ by eliminating the three bracketed negative terms in (43), the following inequality is obtained:

$$
\begin{aligned}
\dot{V} \leq & -k_{3} r_{r}^{T} r_{r}-\alpha_{1} k_{2} K_{I} \tanh ^{2}\left(e_{m}\right)-k_{2} K_{I} \alpha_{3} \tanh ^{2}\left(e_{f}\right) \\
& -\alpha_{2} \eta_{m}^{2}-\left(\alpha_{2}-\frac{\bar{\zeta}_{K}^{2}}{4 \alpha}\right) \eta_{m}^{2}+\frac{\|z\|^{2}}{4 \alpha_{2} k_{n 1}}+\frac{\bar{\zeta}_{K}^{2}\left(x_{m}-x_{r 1}\right)^{2}}{4 \alpha_{2}} .
\end{aligned}
$$

Provided that $k_{n 1}$ is selected according to the sufficient condition

$$
k_{n 1}>\frac{1}{4 \alpha_{2} \min \left\{\alpha_{1} k_{2} \underline{\zeta}_{K}, k_{2} \underline{\zeta}_{K} \alpha_{3}, \alpha_{2}\right\}}
$$

the expression in (44) can be further reduced as follows:

$$
\begin{aligned}
\dot{V} \leq-\lambda_{1}\|z\|^{2}-k_{3}\left\|r_{r}\right\|^{2}- & \left(\alpha_{2}-\frac{\bar{\zeta}_{K}^{2}}{4 \alpha}\right) \\
& \times \eta_{m}^{2}+\frac{\bar{\zeta}_{K}^{2}\left(x_{m}-x_{r 1}\right)^{2}}{4 \alpha_{2}}
\end{aligned}
$$

where $\lambda_{1} \in \mathbb{R}$ is defined as

$$
\lambda_{1}=\min \left\{\alpha_{1} k_{2} \underline{\zeta}_{K}, k_{2} \underline{\zeta}_{K} \alpha_{3}, \alpha_{2}\right\}-\frac{1}{4 \alpha_{2} k_{n 1}} .
$$


Based on (4) in Assumption 1, for the noncontact case

$$
\zeta_{x_{r}} \leq x_{r 1} \leq x_{m} \leq \zeta_{x_{m}} .
$$

Hence, the expression in (45) can be upper bounded as follows:

$$
\dot{V} \leq-\lambda\|y\|^{2}+\varepsilon_{x}
$$

where $\lambda \in \mathbb{R}$ is defined as

$$
\lambda=\min \left\{\lambda_{1}, k_{3},\left(\alpha_{2}-\frac{\bar{\zeta}_{K}^{2}}{4 \alpha}\right)\right\}
$$

and $y(t) \in \mathbb{R}^{5}$ and $\varepsilon_{x} \in \mathbb{R}$ are defined as

$$
y=\left[\begin{array}{ll}
z^{T} & r_{r}^{T}
\end{array}\right]^{T} \quad \varepsilon_{x}=\frac{\bar{\zeta}_{K}^{2}\left(\zeta_{x_{m}}+\left|\zeta_{x_{r}}\right|\right)^{2}}{4 \alpha_{2}}
$$

where $\varepsilon_{x}$ can be made arbitrarily small by making $\alpha_{2}$ large. Based on (39) and (47), either $\lambda\|y\|^{2} \leq \varepsilon_{x}$ or $\lambda\|y\|^{2}>\varepsilon_{x}$. If $\lambda\|y\|^{2}>\varepsilon_{x}$, then Barbalat's lemma [51] can be used to conclude that $\dot{V}(t) \rightarrow 0$ since $V(t)$ is lower bounded, $\dot{V}(t)$ is negative semidefinite, and $\dot{V}(t)$ can be shown to be uniformly continuous. As $\dot{V}(t) \rightarrow 0$, eventually, $\lambda\|y\|^{2} \leq \varepsilon_{x}$. Provided that the sufficient gain condition in (34) is satisfied (i.e., $\varepsilon_{x}<$ $1)$, then (21), (48), and the facts that $\tilde{\theta}_{r}(t)$ and $\tilde{\theta}_{d k}(t) \in \mathcal{L}_{\infty}$ from the use of a projection algorithm can be used to conclude that $V(\cdot) \in \mathcal{L}_{\infty}$; hence, $\|y(t)\|,\|z(t)\|,\left\|r_{r}(t)\right\|,\left\|e_{r}(t)\right\|$, $\eta_{m}(t), e_{f}(t)$, and $e_{m}(t) \in \mathcal{L}_{\infty}$. Signal chasing arguments can be used to prove that the remaining closed-loop signals are also bounded during the noncontact case. The previous development can be used to conclude that for the noncontact case

$$
\|y(t)\| \rightarrow \sqrt{\frac{\varepsilon_{x}}{\lambda}} \text { and, hence, }\left\|r_{r}(t)\right\| \rightarrow \sqrt{\frac{\varepsilon_{x}}{\lambda}} \text { as } t \rightarrow \infty .
$$

Based on (49), linear analysis methods (see Lemma A.19 in [42]) can be applied to (14) to prove that

$$
\left\|e_{r}(t)\right\| \rightarrow\left\|e_{r}(0)\right\| \exp (-\alpha t)+\frac{1}{\alpha} \sqrt{\frac{\varepsilon_{x}}{\lambda}}(1-\exp (-\alpha t))
$$

as $t \rightarrow \infty$ for the noncontact case.

Further analysis is required to prove that the manipulator makes contact with the mass-spring system and to achieve the control objective. Contact between the manipulator and the mass-spring system occurs when $x_{r 1}(t) \geq x_{m}(t)$. Based on (50), a sufficient condition for contact can be developed as follows:

$$
x_{r d 1} \geq x_{m}+\frac{1}{\alpha} \sqrt{\frac{\varepsilon_{x}}{\lambda}} .
$$

After using (22), the sufficient condition in (51) can be expressed as

$Y_{d} \hat{\theta}_{d k}+k_{2} \tanh \left(e_{m}\right)-k_{1} k_{2} \cosh ^{2}\left(e_{f}\right) \tanh \left(e_{f}\right) \geq \frac{1}{\alpha} \sqrt{\frac{\varepsilon_{x}}{\lambda}}$.
By using (13) and (17) and performing some algebraic manipulation, the inequality in (52) can be expressed as

$$
\begin{aligned}
k_{2} \tanh \left(e_{m}\right)-k_{1} k_{2} \cosh ^{2}\left(e_{f}\right) \geq \frac{1}{\alpha} \\
\times \sqrt{\frac{\varepsilon_{x}}{\lambda}}-x_{m d} \underline{\theta}_{d k}+\left(e_{m}+x_{0}\right) \bar{\theta}_{d k}
\end{aligned}
$$

where $\bar{\theta}_{d k}(t)$ and $\underline{\theta}_{d k}(t)$ are defined in (25). From Assumption $1, e_{m}(t)$ can be upper bounded as follows:

$$
e_{m} \leq \bar{\varepsilon}_{m}
$$

where $\bar{\varepsilon}_{m} \in \mathbb{R}$ denotes a known positive constant. If $e_{m}(t) \leq 0$, then the sufficient condition in (53) may not be satisfied. The condition that $e_{m}(t) \leq 0$ will only occur if an impact collision occurs causes the mass to overshoot the desired position. However, even if an impact occurs, and the mass overshoots the desired position, the dynamics will force the mass position error to return to the initial condition. That is, $e_{m}(t) \rightarrow x_{m d}-x_{0}>$ $\underline{\varepsilon}_{m}$, where $\underline{\varepsilon}_{m} \in \mathbb{R}$ denotes a known positive constant. Based on (54) and the fact that $e_{m}(t)$ will eventually be lower bounded by $\underline{\varepsilon}_{m}$ in a noncontact condition, the inequality in (53) can be simplified as follows:

$$
\begin{aligned}
k_{2}\left(\tanh \left(\underline{\varepsilon}_{m}\right)-k_{1}\right. & \left.\cosh ^{2}\left(e_{f}\right)\right) \geq \frac{1}{\alpha} \\
& \times \sqrt{\frac{\varepsilon_{x}}{\lambda}}-x_{m d} \underline{\theta}_{d k}+\left(\bar{\varepsilon}_{m}+x_{0}\right) \bar{\theta}_{d k} .
\end{aligned}
$$

To further simplify the inequality in (55), an upper bound can be determined for $e_{f}(t)$. The inequality in (49) along with (21) and (48) can be used to conclude that as the manipulator approaches the mass, $e_{f}(t)$ will eventually be upper bounded as follows:

$$
e_{f} \leq \tanh ^{-1}\left(\frac{1}{\alpha} \sqrt{\frac{\varepsilon_{x}}{\lambda}}\right) \leq \varepsilon_{f}
$$

where $\varepsilon_{f} \in \mathbb{R}$ is a known positive constant. Based on (49) and (56), the control parameter $k_{2}$ can be selected according to the following sufficient condition to ensure that the robot and the mass-spring system make contact:

$$
k_{2} \geq \frac{\frac{1}{\alpha} \sqrt{\frac{\varepsilon_{x}}{\lambda}}-x_{m d} \underline{\theta}_{d k}+\left(\bar{\varepsilon}_{m}+x_{0}\right) \bar{\theta}_{d k}}{\tanh \left(\underline{\varepsilon}_{m}\right)-k_{1} \cosh ^{2}\left(\varepsilon_{f}\right)}
$$

where $k_{1}$ is chosen as follows:

$$
k_{1}<\frac{\tanh \left(\underline{\varepsilon}_{m}\right)}{\cosh ^{2}\left(\varepsilon_{f}\right)} .
$$

\section{Case 2: Contact}

Provided that the sufficient condition in (57) is satisfied, the robot will eventually make contact with the mass. For the case when the dynamic systems collide $(\Lambda=1)$, and the two 
dynamic systems become coupled, ${ }^{2}$ then (41) can be rewritten as follows:

$$
\begin{aligned}
\dot{V} \leq & -k_{3} r_{r}^{T} r_{r}-\alpha_{1} k_{2} K_{I} \tanh ^{2}\left(e_{m}\right)-3 \alpha_{2} \eta_{m}^{2} \\
& -k_{2} K_{I} \alpha_{3} \tanh ^{2}\left(e_{f}\right)-\left[\alpha\left\|e_{r}\right\|^{2}-\bar{\zeta}_{K}\left|\eta_{m}\right|\left\|e_{r}\right\|\right] \\
& -\left[k_{n 1} \zeta_{1}^{2} \alpha_{2} \eta_{m}^{2}-\zeta_{1}\|z\|\left|\eta_{m}\right|\right]
\end{aligned}
$$

where (20) was substituted for $\chi\left(e_{m}, e_{f}, \eta_{m}, t\right)$. Completing the squares on the last two lines yields

$$
\begin{aligned}
\dot{V} \leq & -k_{3} r_{r}^{T} r_{r}-\alpha_{1} k_{2} K_{I} \tanh ^{2}\left(e_{m}\right) \\
& -3 \alpha_{2} \eta_{m}^{2}-k_{2} K_{I} \alpha_{3} \tanh ^{2}\left(e_{f}\right) \\
& -\left[\alpha\left(\left\|e_{r}\right\|+\frac{\bar{\zeta}_{K}\left|\eta_{m}\right|}{2}\right)^{2}\right]+\frac{\bar{\zeta}_{K}^{2}\left|\eta_{m}\right|^{2}}{4 \alpha} \\
& -\left[k_{n 1} \alpha_{2} \zeta_{1}^{2}\left(\eta_{m}+\frac{\|z\|}{2 k_{n 1} \alpha_{2} \zeta_{1}}\right)^{2}\right]+\frac{\|z\|^{2}}{4 \alpha k_{n 1}} .
\end{aligned}
$$

Eliminating the negative bracketed terms in the last two lines yields

$$
\begin{gathered}
\dot{V} \leq-k_{3} r_{r}^{T} r_{r}-\alpha_{1} k_{2} \underline{\zeta}_{K} \tanh ^{2}\left(e_{m}\right)-\alpha_{3} k_{2} \underline{\zeta}_{K} \tanh ^{2}\left(e_{f}\right) \\
-3 \alpha_{2} \eta_{m}^{2}+\frac{\bar{\zeta}_{K}^{2} \eta_{m}^{2}}{4 \alpha}+\frac{\|z\|^{2}}{4 \alpha_{2} k_{n 1}} \text {. }
\end{gathered}
$$

A final bound can be placed on (58) as follows:

$$
\begin{aligned}
\dot{V} \leq-\min \left\{\alpha_{1} k_{2} \underline{\zeta}_{K}, \alpha_{3} k_{2} \underline{\zeta}_{K}, \alpha_{2}\right\}\|z\|^{2} & \\
& +\frac{\|z\|^{2}}{4 \alpha_{2} k_{n 1}}-\left(2 \alpha_{2}-\frac{\bar{\zeta}_{K}^{2}}{4 \alpha}\right) \eta_{m}^{2}-k_{3} r_{r}^{T} r_{r}
\end{aligned}
$$

Because (39) is nonnegative, and its derivative is negative semidefinite, $r_{r}(t), \tilde{\theta}_{r}(t), \tilde{\theta}_{d k}(t), e_{r}(t), e_{m}(t), e_{f}(t)$, and $\eta_{m}(t) \in \mathcal{L}_{\infty}$. Due to the fact that $e_{m}(t), e_{f}(t)$, and $\eta_{m}(t) \in$ $\mathcal{L}_{\infty}$, the expression in (14) can be used to conclude that $\dot{e}_{m}(t) \in$ $\mathcal{L}_{\infty}$ (and, hence, $e_{m}(t)$ is uniformly continuous). Due to the fact that $e_{m}(t) \in \mathcal{L}_{\infty},(13)$ can be used to conclude that $x_{m}(t) \in$ $\mathcal{L}_{\infty}$. Previous facts can be used to prove that $x_{r d}(t) \in \mathcal{L}_{\infty}$, and, since $e_{r}(t) \in \mathcal{L}_{\infty}$, then $x_{r}(t) \in \mathcal{L}_{\infty}$. Due to the fact that $e_{f}(t)$, $e_{m}(t)$, and $\eta_{m}(t) \in \mathcal{L}_{\infty},(15)$ can be used to conclude that $\dot{e}_{f}(t) \in \mathcal{L}_{\infty}$. The expression in (16) can be used to conclude that $\dot{\eta}_{m}(t) \in \mathcal{L}_{\infty}$ (and, hence, $\eta_{m}(t)$ is uniformly continuous). Given that $r_{r}(t), e_{m}(t), e_{f}(t)$, and $\eta_{m}(t) \in \mathcal{L}_{\infty}, Y_{r}(\cdot) \in \mathcal{L}_{\infty}$. Since $\tilde{\theta}_{r}(t) \in \mathcal{L}_{\infty},(32)$ can be used to prove that $\hat{\theta}_{r}(t) \in \mathcal{L}_{\infty}$. The expression in (29) can then be used to prove that $F(t) \in$ $\mathcal{L}_{\infty}$. The expression in (31) can be used to conclude that $\dot{r}_{r}(t) \in$ $\mathcal{L}_{\infty}$ (and, hence, $r_{r}(t)$ is uniformly continuous). Due to the fact that $e_{m}(t), r_{r}(t)$, and $\eta_{m}(t) \in \mathcal{L}_{2}$ and uniformly continuous, Barbalat's lemma can be used to conclude that $\left|e_{m}(t)\right|,\left\|r_{r}(t)\right\|$, $\left|\eta_{m}(t)\right| \rightarrow 0$ as $t \rightarrow \infty$. Based on the fact that $\left\|r_{r}(t)\right\| \rightarrow 0$ as

\footnotetext{
${ }^{2}$ The dynamic systems can separate after an impact; however, this case can still be analyzed under the noncontact section of the stability analysis.
}

$t \rightarrow \infty$, standard linear analysis methods (see Lemma A.15 in [42]) can then be used to prove that $\left\|e_{r}(t)\right\| \rightarrow 0$ as $t \rightarrow \infty$.

\section{REFERENCES}

[1] O. Khatib, "A unified approach for motion and force control of robot manipulators: The operational space formulation," IEEE J. Robot. Autom., vol. RA-3, no. 1, pp. 43-53, Feb. 1987.

[2] S. Eppinger and W. Seering, "Three dynamic problems in robot force control," in Proc. IEEE Int. Conf. Robot. Autom., May 14-19, 1989, vol. 1, pp. 392-397.

[3] R. Anderson and M. Spong, "Hybrid impedance control of robotic manipulators," in Proc. IEEE Int. Conf. Robot. Autom., Mar. 1987, vol. 4, pp. 1073-1080.

[4] R. Volpe and P. Khosla, "A theoretical and experimental investigation of explicit force control strategies for manipulators," Int. J. Rob. Res., vol. 12 , no. 4, pp. 670-683, Nov. 1994.

[5] D. M. Dawson, F. L. Lewis, and J. F. Dorsey, "Robust force control of a robot manipulator," Int. J. Rob. Res., vol. 11, no. 4, pp. 312-319, Aug. 1992.

[6] M. De Queiroz, J. Hu, D. Dawson, T. Burg, and S. Donepudi, "Adaptive position/force control of robot manipulators without velocity measurements: Theory and experimentation," IEEE Trans. Syst., Man, Cybern. B, Cybern., vol. 27, no. 5, pp. 796-809, Oct. 1997.

[7] S. Hayati, "Hybrid position/force control of multi-arm cooperating robots," in Proc. IEEE Int. Conf. Robot. Autom., Apr. 1986, vol. 3, pp. 82-89.

[8] O. Khatib and J. Burdick, "Motion and force control of robot manipulators," in Proc. IEEE Int. Conf. Robot. Autom., Apr. 1986, vol. 3, pp. 1381-1386.

[9] T. Yoshikawa, "Dynamic hybrid position/force control of robot manipulators description of hand constraints and calculation of joint driving force," in Proc. IEEE Int. Conf. Robot. Autom., Apr. 1986, vol. 3, pp. 1393-1398.

[10] Y.-H. Chen and S. Pandey, "Uncertainty bound-based hybrid control for robot manipulators," IEEE Trans. Robot. Autom., vol. 6, no. 3, pp. 303311, Jun. 1990.

[11] W. Gueaieb, F. Karray, and S. Al-Sharhan, "A robust hybrid intelligent position/force control scheme for cooperative manipulators," IEEE/ASME Trans. Mechatron., vol. 12, no. 2, pp. 109-125, Apr. 2007.

[12] D. Wang and N. McClamroch, "Position and force control for constrained manipulator motion: Lyapunov's direct method," IEEE Trans. Robot. Autom., vol. 9, no. 3, pp. 308-313, Jun. 1993.

[13] L. Whitcomb, S. Arimoto, T. Naniwa, and F. Ozaki, "Experiments in adaptive model-based force control," IEEE Control Syst. Mag., vol. 16, no. 1, pp. 49-57, Feb. 1996.

[14] T. Stepien, L. Sweet, M. Good, and M. Tomizuka, "Control of tool/workpiece contact force with application to robotic deburring," IEEE J. Robot. Autom., vol. RA-3, no. 1, pp. 7-18, Feb. 1987.

[15] Y. Xu, J. Hollerbach, and D. Ma, "A nonlinear PD controller for force and contact transient control," IEEE Control Syst. Mag., vol. 15, no. 1, pp. 15-21, Feb. 1995.

[16] R. Featherstone, "Modeling and control of contact between constrained rigid bodies," IEEE Trans. Robot. Autom., vol. 20, no. 1, pp. 82-92, Feb. 2004.

[17] J. Roy and L. Whitcomb, "Adaptive force control of position/velocity controlled robots: Theory and experiment," IEEE Trans. Robot. Autom., vol. 18, no. 2, pp. 121-137, Apr. 2002.

[18] A. Tornambe, "Modeling and control of impact in mechanical systems: Theory and experimental results," IEEE Trans. Autom. Control, vol. 44, no. 2, pp. 294-309, Feb. 1999.

[19] B. Brogliato, S.-I. Niculescu, and P. Orhant, "On the control of finitedimensional mechanical systems with unilateral constraints," IEEE Trans. Autom. Control, vol. 42, no. 2, pp. 200-215, Feb. 1997.

[20] E. Lee, J. Park, K. Loparo, C. Schrader, and P. H. Chang, "Bang-bang impact control using hybrid impedance/time-delay control," IEEE/ASME Trans. Mechatron., vol. 8, no. 2, pp. 272-277, Jun. 2003.

[21] D. Chiu and S. Lee, "Robust jump impact controller for manipulators," in Proc. IEEE/RSJ Int. Conf. Human Robot Interaction Cooperative Robots, Aug. 1995, pp. 299-304.

[22] P. R. Pagilla and B. Yu, "A stable transition controller for constrained robots," IEEE/ASME Trans. Mechatron., vol. 6, no. 1, pp. 65-74, Mar. 2001.

[23] P. R. Pagilla and B. Yu, "An experimental study of planar impact of a robot manipulator," IEEE/ASME Trans. Mechatron., vol. 9, no. 1, pp. 123-128, Mar. 2004. 
[24] P. Sekhavat, Q. Wu, and N. Sepehri, "Impact control in hydraulic actuators with friction: Theory and experiments," in Proc. IEEE Amer. Controls Conf., Jul. 2004, pp. 4432-4437.

[25] Y. Wu, T.-J. Tarn, N. Xi, and A. Isidori, "On robust impact control via positive acceleration feedback for robot manipulators," in Proc. IEEE Int. Conf. Robot. Autom., Apr. 1996, pp. 1891-1896.

[26] M. Indri and A. Tornambe, "Impact model and control of two multi-DOF cooperating manipulators," IEEE Trans. Autom. Control, vol. 44, no. 6, pp. 1297-1303, Jun. 1999.

[27] A. Tornambe, "Global regulation of a planar robot arm striking a surface," IEEE Trans. Autom. Control, vol. 41, no. 10, pp. 1517-1521, Oct. 1996.

[28] S. P. DiMaio and S. E. Salcudean, "Needle insertion modeling and simulation," IEEE Trans. Robot. Autom., vol. 19, no. 5, pp. 864-875, Oct. 2003.

[29] A. M. Okamura, C. Simone, and M. D. O'Leary, "Force modeling for needle insertion into soft tissue," IEEE Trans. Biomed. Eng., vol. 51, no. 10 , pp. $1707-1716$, Oct. 2004.

[30] M. P. Ottensmeyer and J. K. Salisbury, "In vivo data acquisition instruments for solid organ mechanical property measurement," in Proc. Int. Conf. Med. Image Comput. Comput.-Assisted Intervention, Oct. 2001, pp. 975-982.

[31] F. S. Azar, D. N. Metaxas, and M. D. Schnall, "A finite element model of the breast for predicting mechanical deformations during biopsy procedures," in Proc. IEEE Workshop Math. Methods Biomed. Image Anal., Jun. 2000, pp. 38-45.

[32] Y. C. Fung, Biomechanics: Mechanical Properties of Living Tissue, 2nd ed. New York: Springer-Verlag, 1993.

[33] K. Dupree, C. Liang, G. Hu, and W. E. Dixon, "Global adaptive Lyapunov-based control of a robot and mass-spring system undergoing an impact collision," in Proc. IEEE Conf. Decision Control, Dec. 2006, pp. 2039-2044.

[34] G. Hu, W. E. Dixon, and C. Makkar, "Energy-based nonlinear control of underactuated Euler-Lagrange systems subject to impacts," in Proc. IEEE Conf. Decision Control, Dec. 2005, pp. 6859-6864.

[35] G. Hu, W. E. Dixon, and C. Makkar, "Energy-based nonlinear control of underactuated Euler-Lagrange systems subject to impacts," IEEE Trans. Autom. Control, vol. 52, no. 9, pp. 1742-1748, Sep. 2007.

[36] M. Indri and A. Tornambe, "Control of under-actuated mechanical systems subject to smooth impacts," in Proc. IEEE Conf. Decision Control, Dec. 2004, pp. 1228-1233.

[37] M. Krstic, P. V. Kokotovic, and I. Kanellakopoulos, Nonlinear and Adaptive Control Design. New York: Wiley, 1995.

[38] F. L. Lewis, C. T. Abdallah, and D. M. Dawson, Control of Robot Manipulators, J. Griffin, Ed. New York: Macmillan, 1993.

[39] M. W. Spong, S. Hutchinson, and M. Vidyasagar, Robot Modeling and Control. Hoboken, NJ: Wiley, 2006.

[40] W. E. Dixon, E. Zergeroglu, D. M. Dawson, and M. W. Hannan, "Global adaptive partial state feedback tracking control of rigid-link flexible-joint robots," in Proc. IEEE/ASME Int. Conf. Advanced Intell. Mechatronics, Sep. 1999 , pp. 281-286.

[41] F. Zhang, D. M. Dawson, M. S. de Queiroz, and W. E. Dixon, "Global adaptive output feedback tracking control of robot manipulators," IEEE Trans. Autom. Control, vol. 45, no. 6, pp. 1203-1208, Jun. 2000.

[42] W. E. Dixon, A. Behal, D. M. Dawson, and S. Nagarkatti, Nonlinear Control of Engineering Systems: A Lyapunov-Based Approach. Boston, MA: Birkhäuser, 2003.

[43] Z. Cai, M. S. de Queiroz, and D. M. Dawson, "A sufficiently smooth projection operator," IEEE Trans. Autom. Control, vol. 51, no. 1, pp. 135139, Jan. 2006.

[44] B. Brogliato and P. Orhant, "Contact stability analysis of a one degree-offreedom robot," Dyn. Control, vol. 8, no. 1, pp. 37-53, Jan. 1998.

[45] M. S. Loffler, N. P. Costescu, and D. M. Dawson, "QMotor 3.0 and the QMotor robotic toolkit: A PC-based control platform," IEEE Control Syst. Mag., vol. 22, no. 3, pp. 12-26, Jun. 2002.

[46] N. Stefanovic, M. Ding, and L. Pavel, "An application of $L_{2}$ nonlinear control and gain scheduling to erbium doped fiber amplifiers," Control Eng. Pract., vol. 15, no. 9, pp. 1107-1117, Sep. 2007.

[47] T. Fujinaka, Y. Kishida, M. Yoshioka, and S. Omatu, "Stabilization of double inverted pendulum with self-tuning neuro-PID," in Proc. IEEE/INNS/ENNS Int. Conf. Neural Netw., Jul. 24-27, 2000, vol. 4, pp. 345-348.

[48] F. Nagata, K. Kuribayashi, K. Kiguchi, and K. Watanabe, "Simulation of fine gain tuning using genetic algorithms for model-based robotic servo controllers," in Proc. Int. Symp. Comput. Intell. Robot. Autom., Jun. 20-23, 2007, pp. 196-201.

[49] R. Kelly, V. Santibanez, and A. Loria, Control of Robot Manipulators in Joint Space. New York: Springer-Verlag, 2005.
[50] [Online]. Available: http://ncr.mae.ufl.edu/projects/robman/ adaptiveimpact.htm

[51] J. J. Slotine and W. Le, Applied Nonlinear Control. Englewood Cliffs, NJ: Prentice-Hall, 1991.

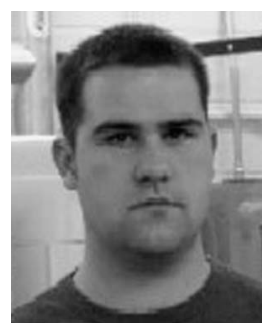

Keith Dupree (S'06) received the B.S. degree in aerospace engineering and the M.S. degree in mechanical engineering from the University of Florida, Gainesville, in 2005 and 2007, respectively. He is currently working toward the Ph.D. degree at the Department of Mechanical and Aerospace Engineering, University of Florida.

$\mathrm{He}$ is a member of the Nonlinear Controls and Robotics Group, Department of Mechanical and Aerospace Engineering, University of Florida. His research interests include optimal control, nonlinear control, and visual servo control.

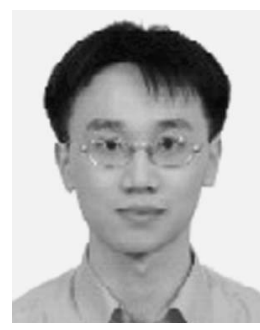

Chien-Hao Liang (S'06) received the B.S. degree in ocean engineering from the National Taiwan University, Taipei, Taiwan, R.O.C., in 2001 and the M.S degree in mechanical engineering from the University of Florida, Gainesville, in 2007. His master's thesis focused on impact control strategies using Lyapunov-based control methods.

His research interests include robot motion control, man-machine interaction control, and robot manipulator control.

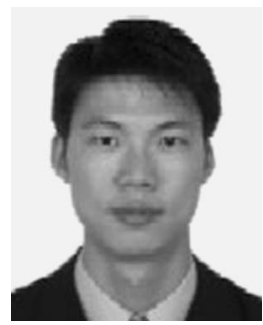

Guoqiang Hu (S'05-M'08) received the B.Eng. degree from the University of Science and Technology of China, Hefei, China, in 2002, the M.Phil. degree from the Chinese University of Hong Kong, Shatin, Hong Kong, in 2004, and the Ph.D. degree from the University of Florida, Gainesville, in 2007.

After finishing his postdoctoral research at the University of Florida, he joined the Department of Mechanical and Nuclear Engineering, Kansas State University, Manhattan, as an Assistant Professor. His main research interests include vision-based control and state estimation, and nonlinear and adaptive control of dynamic systems.

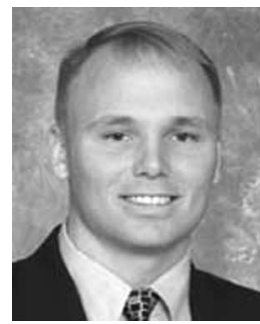

Warren E. Dixon (S'94-M'00-SM'05) received the $\mathrm{Ph} . \mathrm{D}$. degree from Clemson University, Clemson, SC, in 2000.

After completing his doctoral studies, he was selected as a Eugene P. Wigner Fellow at Oak Ridge National Laboratory, Oak Ridge, TN. In 2004, he joined the faculty of the Department of Mechanical and Aerospace Engineering, University of Florida, Gainesville. He has published two books and over 150 journal and conference papers on the development and application of Lyapunov-based control

methods

Dr. Dixon is a member of numerous conference program committees, technical committees, organizing committees, and conference editorial boards $\mathrm{He}$ is an appointed member of the IEEE Control Systems Society Board of Governors and is currently an Associate Editor for the IEEE TRANSACTIONS ON Systems, MAN, AND CYBERnetics: PART B CYBERnETICS. His efforts in this area have been acknowledged through awards such as a National Science Foundation CAREER award and the IEEE Robotics and Automation Society Early Academic Career Award. 